\section{(6) OPEN ACCESS}

\title{
Identification of myeloid cells in the human enthesis as the main source of local IL-23 production
}

\author{
Charlie Bridgewood, ${ }^{01}$ Abdulla Watad, ${ }_{1}^{1,2,3}$ Tobias Russell, ${ }^{1}$ Timothy M Palmer, ${ }^{4}$ \\ Helena Marzo-Ortega ${ }_{1}^{1,5}$ Almas Khan, ${ }^{6}$ Peter A Millner, ${ }^{6}$ Robert Dunsmuir, ${ }^{6}$ \\ Abhay Rao, ${ }^{6}$ Peter Loughenbury, ${ }^{6}$ Miriam Wittmann, ${ }^{1,5}$ Richard J Cuthbert, ${ }^{1}$ \\ Dennis G McGonagle 1,5
}

\section{Handling editor Josef $S$ \\ Smolen}

- Additional material is published online only. To view please visit the journal online (http://dx.doi.org/10.1136/ annrheumdis-2018-214944)

${ }^{1}$ Leeds Institute of Rheumatic and Musculoskeletal Medicine (LIRMM), University of Leeds, Leeds, UK and Zabludowicz Center for Autoimmune Diseases, Sheba Medical Center, Tel Aviv, Israel ${ }^{3}$ Sackler Faculty of Medicine, Tel Aviv University, Tel Aviv, Israel ${ }^{4}$ Centre for Atherothrombosis and Metabolic Disease, Hull

York Medical School, University of Hull, Hull, UK

${ }^{5}$ National Institute for Biomedical Research Centre (BRC), Leeds Teaching Hospitals, Leeds, UK

${ }^{6}$ Leeds Teaching Hospitals NHS Trust, Leeds, UK

\section{Correspondence to} Prof Dennis G McGonagle, Leeds Institute of Rheumatic and Musculoskeletal Medicine, University of Leeds, Leeds, United Kingdom; d.g.mcgonagle@leeds.ac.uk

Received 18 December 2018 Revised 19 March 2019 Accepted 23 March 2019 24 April 2019
${ }^{2}$ Department of Medicine 'B' HealthResearch (NIHR) Leeds Published Online First

\section{ABSTRACT}

Objective We investigated whether the normal human spinal enthesis contained resident myeloid cell populations, capable of producing pivotal proinflammatory cytokines including tumour necrosis factor (TNF) and interleukin (IL)-23 and determined whether these could be modified by PDE4 inhibition. Methods Normal human enthesis soft tissue (ST) and adjacent perientheseal bone (PEB) $(n=15)$ were evaluated using immunohistochemistry $(\mathrm{IHC})$, digested for myeloid cell phenotyping, sorted and stimulated with different adjuvants (lipopolysaccharide and mannan). Stimulated enthesis fractions were analysed for inducible production of spondyloarthropathy disease-relevant mediators (IL-23 full protein, TNF, IL-1 $\beta$ and CCL20). Myeloid populations were also compared with matched blood populations for further mRNA analysis and the effect of PDE4 inhibition was assessed.

Results A myeloid cell population (CD45+ HLADR+ CD14+ CD11c+) phenotype was isolated from both the ST and adjacent PEB and termed 'CD14+ myeloid cells' with tissue localisation confirmed by CD14+ IHC. The CD14- fraction contained a CD123+ HLADR+ CD11c - cell population (plasmacytoid dendritic cells). The CD14+ population was the dominant entheseal producer of IL-23, IL-1 $\beta$, TNF and CCL20. IL-23 and TNF from the CD14+ population could be downregulated by a PDE4I and other agents (histamine and 8-Bromo-CAMP) which elevate CAMP. Entheseal CD14+ cells had a broadly similar gene expression profile to the corresponding CD14+ population from matched blood but showed significantly lower CCR2 gene expression.

Conclusions The human enthesis contains a CD14+ myeloid population that produces most of the inducible IL-23, IL-1 $\beta$, TNF and CCL20. This population has similar gene expression profile to the matched blood CD14+ population.

\section{Check for updates}

(c) Author(s) (or their employer(s)) 2019. Re-use permitted under CC BY-NC. No commercial re-use. See rights and permissions. Published by BMJ.

To cite: Bridgewood $C$

Watad A, Russell T,

et al. Ann Rheum Dis

2019;78:929-933.

\section{INTRODUCTION}

Synovitis is the primary lesion in rheumatoid arthritis (RA), whereas in spondyloarthropathy $(\mathrm{SpA})$-related diseases, enthesitis is the cardinal lesion. ${ }^{1}$ Psoriatic arthritis (PsA) and the associated $\mathrm{SpA}$ disorders are linked to multiple genetic polymorphisms in the interleukin (IL)-23/17 axis. Manipulation of this pathway results in an enthesitis-dependent murine arthritis and several populations of IL-23R positive cells reside at the normal

\section{Key messages}

What is already known about this subject?

- Interleukin (IL)-23-producing myeloid cells are thought to be key to the pathogenesis of closely related disease such as psoriasis and inflammatory bowel disease.

- The enthesis is considered the cardinal lesion in psoriatic arthritis (PsA) and spondyloarthropathy (SpA) but it was previously unknown if this tissue contained a population of myeloid cells capable of IL-23 production.

- It has been considered that intestinal or systemically derived IL-23 may have been responsible for triggering enthesitis.

What does this study add?

- We demonstrate that normal spinal enthesis soft tissue and bone has resident myeloid cells.

- It was possible to induce IL-23 protein which was almost exclusive to a population of CD14+ myeloid cells.

How might this impact on clinical practice or future developments?

- This study increases the understanding of innate immunity at the normal healthy human enthesis that needs further study to understand how this might contribute to blocking IL-23 efficacy, or not in some settings, in PsA and SpA.

murine and human enthesis. ${ }^{1-4}$ The observed difference in efficacy of IL-23/17 axis blockers and phosphodiesterase 4 inhibitor (PDE4I) therapy in RA and SpA may be a result of immunopathogenic differences linked to the location of the primary site of inflammation. ${ }^{156}$

The prevailing theories have proposed that the IL-23 driving enthesitis originates at remote sites including the gut. $^{3}$ In both human psoriasis and mouse models of psoriasis, the major producers of IL-23 are skin resident myeloid cells. ${ }^{7}$ Studies also implicate myeloid cells in human enthesitis with macrophages being present in early SpA-related enthesitis. $^{8}$ A recent mouse model of mechanical strain also confirmed enthesitis was dependent on myeloid infiltration. ${ }^{9}$

Accordingly, we hypothesised that the human enthesis harbours a population of myeloid cells 
capable of proinflammatory cytokine production. We investigated whether potential IL-23 secretion could be modulated by PDE4 inhibition, a target pathway known to show efficacy in PsA but not RA.

\section{MATERIALS AND METHODS}

Comprehensive materials and methods can be found in online supplementary materials. Briefly, for tissue collection, normal spinous process enthesis was obtained from 15 patients (6 men and 9 women; median age 46 years) who were undergoing spinal decompression or surgery for scoliosis correction of thoracic or lumbar vertebrae using previously described methods ${ }^{4}$ (see online supplementary figure 1). The study protocol of the current investigation was approved by North West-Greater Manchester West Research Ethics Committee.

\section{Statistical analysis}

Statistical significance was calculated using a one or two-way analysis of variance with Bonferroni's multiple comparisons test, unless stated. Analysis was performed using Prism software (GraphPad software V.8.0, La Jolla, CA, USA). Error bars represent the SEM.

\section{RESULTS}

\section{Myeloid cells are present in entheseal soft tissue and bone} and can produce IL-23

Following the digestion of both the perientheseal bone (PEB) and soft tissue (ST) enthesis and flow cytometry analysis, a myeloid $\mathrm{CD} 45+\mathrm{CD} 14+\mathrm{HLADR}+\mathrm{CD} 11 \mathrm{c}+$ (hereafter referred to as the CD14+ population) and a CD45+ CD14- (hereafter referred to as the CD14- population) of cells were evident (see online supplementary figures 2-4). Within the CD14- fraction, a CD123 + HLADR + CD11c- (plasmacytoid dendritic cell) population was identified, accounting for 1\% of CD $45+$ cells (online supplementary figure 2). Due to demands of using multiple colours for flow cytometry, we decided to exclude the analysis of ILC3 cells, which we have previously identified at the human enthesis. ${ }^{4}$ Histological staining of both the PEB and ST showed CD14+ enthesis immune cell populations (figure 1A, B). For control staining and isotype control, see online supplementary figure 5 .

Following the isolation of CD14+ and CD14- fractions by fluorescence-activated cell sorting (FACS), they were stimulated with either mannan or lipopolysaccharide (LPS) and interferon gamma (IFN $\gamma)$. Disease-relevant cytokines and chemokines, tumour necrosis factor (TNF), IL-23 (full protein $\mathrm{p} 40+\mathrm{p} 19$ ), CCL2 0 and IL-1 $\beta$ were measured by ELISA (figure 1C-J). IL-23 and CCL20 were only inducible from CD14+ cells (red box plots) following stimulation (figure $1 \mathrm{E}-\mathrm{H}$ ). While both fractions CD14+ and CD14- showed inducible TNF and IL-1 $\beta$ following stimulation, this was only statistically significant in the CD14+ fraction (figure 1C, D, I, J).

In terms of weight, the PEB contained more CD14+ per gram than the ST (mean $=7.9 \times 10^{4}$ vs $\left.6.8 \times 10^{3}\right)$. Because of the scarcity of available cells in the ST, CD14+ and CD14 - cells were seeded at a lower density than those from the bone (see the Materials and methods section). The individual CD14 + myeloid cell production of IL-23 and TNF expressed as pg/cell was not significantly different between PEB and ST (online supplementary figure 6). Intracellular staining for IL-23 of both CD14+ and CD14- populations
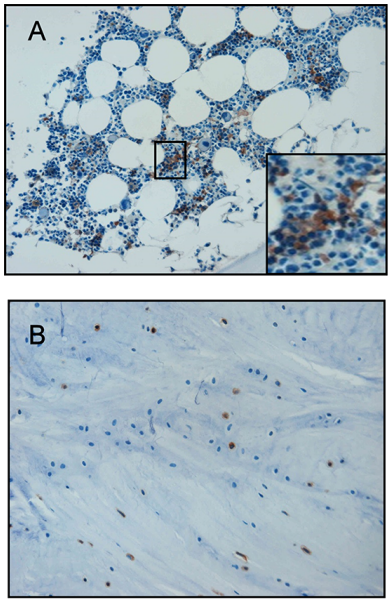

K

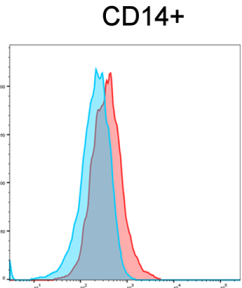

IL-23p19
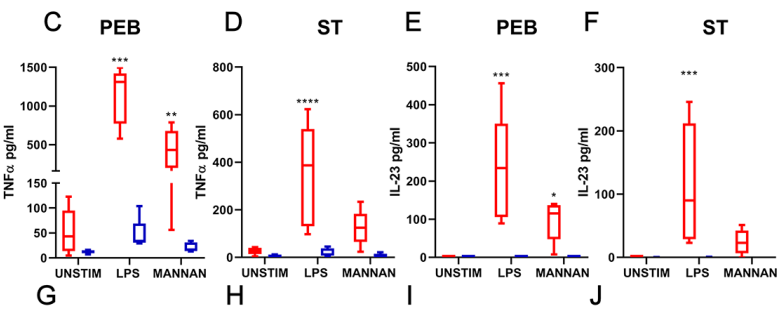

PEB
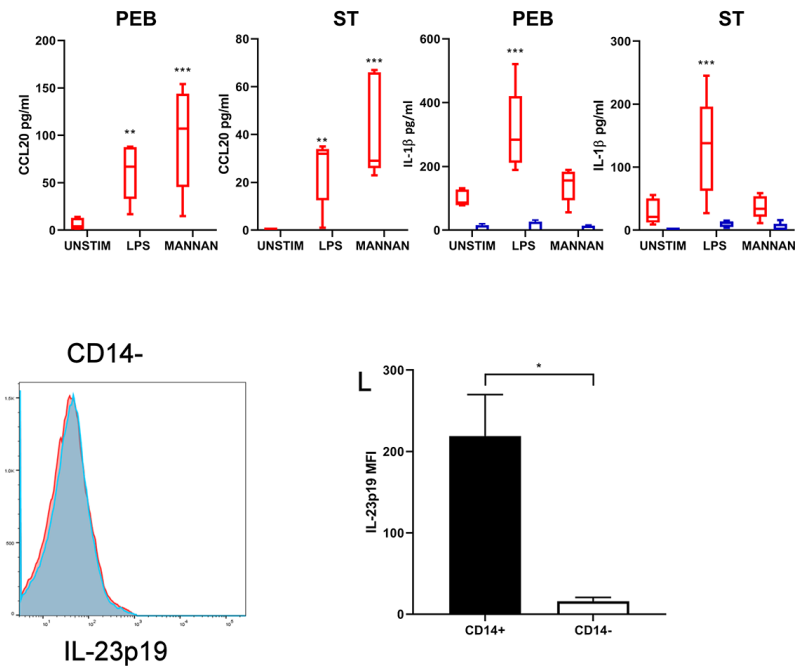

Figure 1 Immunohistochemistry of CD14+ cells in (A) perientheseal bone (PEB) and (B) entheseal soft tissue (ST). Enthesis samples were separated into PEB and ST and CD14+ and CD14- cells sorted. (C-J) CD14+ and CD14- fractions were stimulated with LPS/interferon gamma (IFNY) or mannan for 48 hours. TNF, IL-23, CCL20 and IL-1 $\beta$ levels were measured by ELISA. $n=5$. Two-way analysis of variance (ANOVA) with Bonferroni multiple comparisons test was performed. *Significance from relevant unstimulated control, for example, CD14+ or CD14- unstimulated. (K) PEB was stimulated with LPS/IFNY in the presence of Golgi plug for 16 hours. Cells were surface stained for CD14+ and CD14- cells and intracellular IL-23p19 were measured. Blue histograms represent unstimulated and red stimulated. (L) Change in IL-23p19 median fluorescence intensity (MFI), following LPS/IFNY stimulation in both CD14+ and CD14- gates. $n=3$. Paired t-test. ${ }^{*} p<0.05 ;{ }^{* *} p<0.01 ;{ }^{* *} p<0.001$. IL, interleukin; LPS, lipopolysaccharide; PEB, perientheseal bone; ST, soft tissue; TNF- $\alpha$, tumour necrosis factor alpha. 


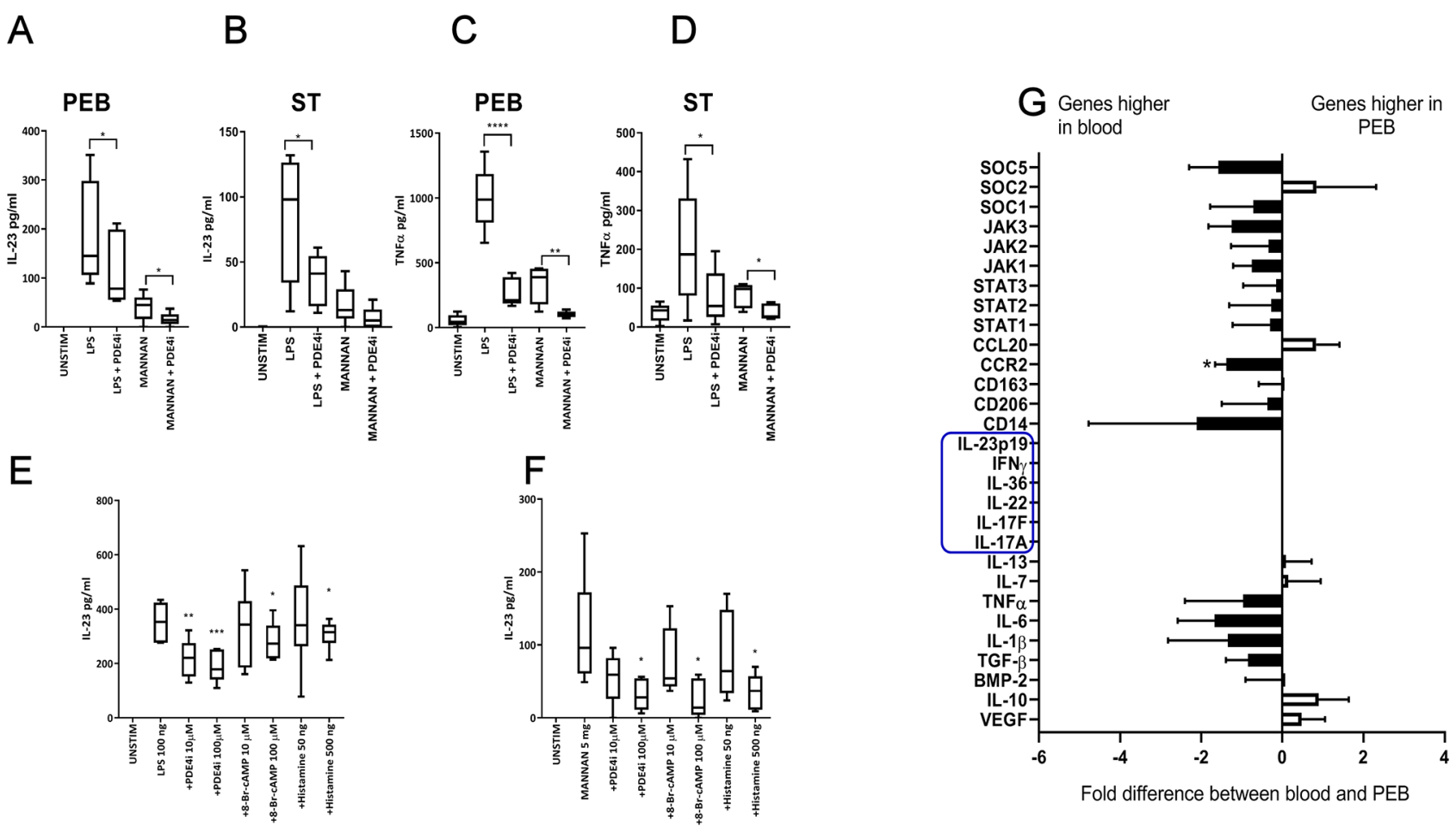

Figure 2 (A-D) CD14+ fraction cells were isolated from the PEB and ST as before $(n=5)$, and stimulated with LPS/IFNY and mannan as before, with and without PDE4I rolipram, IL-23 and TNF were measured by ELISA. (E, F) Healthy blood CD14+ cells were stimulated with LPS/IFNY (E) or mannan (F) with and without CAMP-elevating agents; rolipram, 8-Br-CAMP and histamine for 48 hours $(n=7)$, IL-23 secretion was measured by ELISA. Oneway analysis of variance (ANOVA) with Bonferroni multiple comparisons test was performed. (E, F) Significance from stimulated (LPS or mannan). (G) CD14+ cells were isolated from the PEB or matched blood and transcript analysis was performed $(n=4)$. Black bars represent those higher expressed in blood, white bars those with higher expression in PEB. Genes highlighted in blue were undetectable in both blood and PEB. A paired t-test was performed on $\triangle C$ t values for PEB versus blood. ${ }^{*} p<0.05 ;{ }^{* *} p<0.01 ;{ }^{* *} p<0.001$. IFN, interferon; IL, interleukin; LPS, lipopolysaccharide; PEB, perientheseal bone; ST, soft tissue; TNF, tumour necrosis factor; VEGF, vascular endothelial growth factor.

following LPS/IFN $\gamma$ stimulation also confirmed CD14+ as the exclusive IL-23 producer in the PEB (figure $1 \mathrm{~K}, \mathrm{~L}$ ).

\section{PDE4I and other CAMP-elevating agents inhibit IL-23 from CD14+ entheseal and blood myeloid cells}

From figure 1, it was evident that CD14+ cells were the exclusive IL-23 producers. We next tested the ability of a PDE4I (rolipram) to modulate IL-23 secretion. Rolipram is widely used in vitro to study the effects of PDE4 inhibition and has a similar profile to apremilast. ${ }^{10}$ Blockade of the PDE4 pathway is efficacious in PsA but not RA. Rolipram effectively inhibited both mannan/IFN $\gamma$ and LPS/IFN $\gamma$-induced IL-23 and TNF from the CD14+ fraction from both ST and PEB (figure 2A-D).

PDE4Is are thought to modulate inflammatory pathways via elevating cAMP. Due to the low number of cells yielded from the enthesis, cAMP pathway exploratory research on IL-23 secretion was conducted on the corresponding CD14+ population in blood from healthy donors. CD14+ cells were isolated by FACS and stimulated with LPS/IFN $\gamma$ (figure 2E) or mannan/IFN $\gamma$ (figure $2 \mathrm{~F}$ ) as before. To access the importance of the cAMP pathway in PDE4I attenuation of IL-23 production, other molecules known to activate the cAMP pathway (histamine and 8-Bromo-cAMP) were tested for their ability to inhibit IL-23 secretion and confirm the importance of the PDE4 pathway on these cells (figure 2E, F).

\section{Entheseal myeloid cells share a similar gene profile to matched blood cells}

In order to provide further functional characterisation of CD14+ myeloid cells in the enthesis, entheseal cells were compared with those isolated from matched blood (figure $2 \mathrm{G}$ ).
Both populations had a broadly similar gene expression profile. The majority of genes analysed did not have a greater than twofold difference and only CCR2 expression was statistically significant (figure 2G). However, the majority of inflammatory cytokines and inflammatory signalling pathways trended higher in the blood population.

\section{DISCUSSION}

In this study, we report that both normal entheseal ST and adjacent bone have a population of CD45+ CD14+ HLDR+ CD11c+ myeloid cells. Entheseal CD14+ cells are capable of producing IL-23, TNF, IL-1 $\beta$ and CCL20. Moreover, IL-23 and TNF induction can be reduced by PDE4 inhibition. These observations may also be of relevance to disease, given that both PDE4 inhibitors and IL-23 blockers show efficacy in PsA but not RA.

In a landmark IL-23-dependent enthesitis mouse model of SpA, it was shown that in vivo hepatic expression of IL-23 induced enthesitis via an effect on resident enthesis $\mathrm{T}$ cells and it was considered that the IL-23 effect was via systemically released cytokine. ${ }^{3}$ Presently, there are no reports of normal enthesis myeloid cells with the ability to secrete IL-23 locally. Herein we show that cells capable of producing IL-23 are present at the enthesis and that virtually all of the IL-23 was from the CD14+ fraction as opposed to the CD14- fraction. This highlights the potential importance of this myeloid cell population in disease initiation. Both bacterial and fungal adjuvants were able to induce IL-23 production from the CD14+ fraction. In agreement with this, in the SKG mouse model of arthritis where pathology originates as enthesitis, both curdlan and chlamydia promote disease. ${ }^{11} 12$ Moreover, previous 
research has shown myeloid cells such as macrophages in the early inflamed SpA enthesis. ${ }^{8}$

It was previously unknown if IL-23-producing myeloid cells at the enthesis were resident or tissue infiltrating. Classification of myeloid cell subsets within tissues is notoriously difficult and remains controversial. A new single-cell RNA-seq approach may be the only way to truly define the myeloid subsets. ${ }^{13}$ During inflammation, classical monocytes (defined as CD14high CD16low) enter tissues in a CCR2-CCL2 dependent manner. In a recent mouse model of biomechanical loading, arthritis was dependent on CCL2 production and subsequent monocyte recruitment. ${ }^{9}$ The myeloid cells we described at the enthesis may be non-classical monocytes. It is known that non-classical monocytes migrate and 'patrol' peripheral tissues, so are not defined as tissue resident macrophages in the sense of microglial or Kupffer cells. ${ }^{14}$ Non-classical monocytes (defined as CD14low CD16high) are known to express less CCR2 and inflammatory mediators when compared with classical monocytes, which would align with our findings. ${ }^{14}$ Entheseal myeloid cells in our study indeed showed statistically significantly less expression of CCR2, while basal production of inflammatory mediators such as IL-1 $\beta$, IL-6 and TNF trended downwards.

We also report that fungal and bacterial adjuvants are able to induce CCL20 from enthesis CD14 + cells, but not CD14- cells. CCL20 is a chemokine that attracts IL-17-expressing cells via its ligand CCR6. In agreement with this, PsA synovial fluid levels of CCL20 correlate with disease severity. ${ }^{15}$ IL-17-expressing ILC3 and $\mathrm{T}$ cells are also CCR6 + from the synovial fluid of patients with PsA. ${ }^{16}{ }^{17}$ These findings suggest a link between enthesis myeloid cells and the cells that preferentially accumulate in the adjacent joint cavity in PsA.

While therapeutic blockage of IL-23 has demonstrated efficacy in PsA, recent results from a clinical trial in patients with ankylosing spondylitis have proved disappointing. ${ }^{18}$ It has been hypothesised that variation in efficacy may be due to a differing immunopathogenesis including different immune cell populations between the spine and peripheral joint. ${ }^{19} \mathrm{~A}$ recent paper using an HLA-B27 rat model showed that SpA pathology is dependent on IL-23 for initiation but not disease persistence. ${ }^{20}$ This may indeed point towards the idea of IL-17 independent of IL-23 for disease progression, while IL-23 is required for disease initiation. Related research has shown that torn supraspinatus tendon contains IL-17+ cells including T cells; however, it is not known if these cells can produce IL-17 independently of IL-23. ${ }^{21}$

PDE4Is are thought to mediate anti-inflammatory effects by elevating cAMP. Other cAMP pathway activators such as histamine and 8-Bromo-cAMP are known to attenuate inflammatory cytokine production from peripheral blood mononuclear cells; however, this is the first report showing this on tissue myeloid cells. $^{22}$ Previous research has suggested cAMP-elevating agents downregulate IL-12p40, the common chain between IL-12 and IL-23, which in theory may also account for the downregulation of full IL-23 protein $(\mathrm{p} 19+\mathrm{p} 40)$ observed (figure 2A-D). ${ }^{22}$ Further work is required to decipher the exact mechanisms in which cAMP elevation regulates individual IL-12 family member chains.

In conclusion, these data demonstrate that the normal enthesis contains a population of myeloid cells capable of local innate cytokine production including TNF and IL-23. While CD14+ HLADR + CD11c + confirm the presence of a myeloid phenotype, further work is required to fully characterise this population. The in vivo triggers of myeloid-derived TNF and IL-23 remain to be fully elucidated but our findings show that the normal enthesis contains myeloid cells capable of locally contributing to disease.
Acknowledgements The authors thank Abed El Rahman Wattad for providing the online supplementary figure 1. This article/paper/report presentsindependent research funded/supported by the National Institute for HealthResearch (NIHR) Leeds Biomedical Research Centre (BRC). The views expressed arethose of the author(s) and not necessarily those of the NIHR or the Departmentof Health and Social Care.

Contributors $C B, A W, T P, T R, M W, R J C$ and DGM contributed to the acquisition, analysis or interpretation of data and critical revision of the manuscript. AK, HMO $\mathrm{PAM}, \mathrm{RD}, \mathrm{AB}$ and $\mathrm{PL}$ contributed to the acquisition of data and drafting of the manuscript.

Funding The authors have not declared a specific grant for this research from any funding agency in the public, commercial or not-for-profit sectors.

Competing interests None declared.

Patient consent for publication Not required.

Ethics approval The current study has been approved by the University of Leeds ethical approval committee.

Provenance and peer review Not commissioned; externally peer reviewed.

Data sharing statement All data relevant to the study are included in the article or uploaded as supplementary information.

Open access This is an open access article distributed in accordance with the Creative Commons Attribution Non Commercial (CC BY-NC 4.0) license, which permits others to distribute, remix, adapt, build upon this work non-commercially, and license their derivative works on different terms, provided the original work is properly cited, appropriate credit is given, any changes made indicated, and the use is non-commercial. See: http://creativecommons.org/licenses/by-nc/4.0/.

\section{REFERENCES}

1 Bridgewood C, Watad A, Cuthbert RJ, et al. Spondyloarthritis: new insights into clinical aspects, translational immunology and therapeutics. Curr Opin Rheumatol 2018;30.

2 Reveille JD, Sims A-M, Danoy P, et al. Genome-wide association study of ankylosing spondylitis identifies non-MHC susceptibility loci. Nat Genet 2010;42:123-7.

3 Sherlock JP, Joyce-Shaikh B, Turner SP, et al. IL-23 induces spondyloarthropathy by acting on ROR- $\gamma t+$ CD3+CD4-CD8- entheseal resident T cells. Nat Med 2012;18:1069-76.

4 Cuthbert RJ, Fragkakis EM, Dunsmuir R, et al. Brief report: group 3 innate lymphoid cells in human Enthesis. Arthritis Rheumatol 2017;69:1816-22.

5 Smolen JS, Agarwal SK, llivanova E, et al. A randomised phase II study evaluating the efficacy and safety of subcutaneously administered ustekinumab and guselkumab in patients with active rheumatoid arthritis despite treatment with methotrexate. Ann Rheum Dis 2017;76:831-9.

6 Genovese MC, Jarosova K, Cieślak D, et al. Apremilast in patients with active rheumatoid arthritis: a phase II, multicenter, randomized, double-blind, placebocontrolled, parallel-group study. Arthritis Rheumatol 2015;67:1703-10.

7 Yawalkar N, Tscharner GG, Hunger RE, et al. Increased expression of IL-12p70 and IL23 by multiple dendritic cell and macrophage subsets in plaque psoriasis. J Dermatol Sci 2009;54:99-105.

8 McGonagle D, Marzo-Ortega $\mathrm{H}, \mathrm{O}^{\prime}$ Connor $\mathrm{P}$, et al. Histological assessment of the early enthesitis lesion in spondyloarthropathy. Ann Rheum Dis 2002;61:534-7.

9 Cambré I, Gaublomme D, Burssens A, et al. Mechanical strain determines the sitespecific localization of inflammation and tissue damage in arthritis. Nat Commun 2018;9.

10 Schafer PH, Parton A, Capone L, et al. Apremilast is a selective PDE4 inhibitor with regulatory effects on innate immunity. Cell Signal 2014;26:2016-29.

11 Ruutu M, Thomas G, Steck R, et al. $\beta$-glucan triggers spondylarthritis and Crohn's disease-like ileitis in SKG mice. Arthritis Rheum 2012;64:2211-22.

12 Baillet AC, Rehaume LM, Benham H, et al. High Chlamydia Burden promotes tumor necrosis factor-dependent reactive arthritis in SKG Mice. Arthritis Rheumatol 2015;67:1535-47.

13 Villani A-C, Satija R, Reynolds G, et al. Single-cell RNA-seq reveals new types of human blood dendritic cells, monocytes, and progenitors. Science 2017;356 doi:10.1126/science.aah4573

14 Auffray C, Fogg D, Garfa M, et al. Monitoring of blood vessels and tissues by a population of monocytes with patrolling behavior. Science 2007;317:666-70.

15 Celis R, Planell N, Fernández-Sueiro JL, et al. Synovial cytokine expression in psoriatic arthritis and associations with lymphoid neogenesis and clinical features. Arthritis Res Ther 2012:14:R93-93.

16 Benham H, Norris P, Goodall J, et al. Th17 and Th22 cells in psoriatic arthritis and psoriasis. Arthritis Res Ther 2013;15.

17 Leijten EFA, van Kempen TS, Boes M, et al. Brief report: enrichment of activated group 3 innate lymphoid cells in psoriatic arthritis synovial fluid. Arthritis Rheumatol 2015;67:2673-8

18 Baeten D, Østergaard M, Wei JC-C, et al. Risankizumab, an IL-23 inhibitor, for ankylosing spondylitis: results of a randomised, double-blind, placebo-controlled, proof-of-concept, dose-finding phase 2 study. Ann Rheum Dis 2018;77:1295-302. 
19 Siebert S, Millar NL, McInnes IB. Why did IL-23p19 inhibition fail in as: a tale of tissues, trials or translation? Ann Rheum Dis 2018. doi:10.1136/ annrheumdis-2018-213654

20 van Tok MN, Na S, Lao CR, et al. The initiation, but not the persistence, of experimental spondyloarthritis is dependent on interleukin-23 signaling. Front Immunol 2018;9.
21 Millar NL, Akbar M, Campbell AL, et al. IL-17A mediates inflammatory and tissue remodelling events in early human tendinopathy. Sci Rep 2016;6.

22 Kalim KW, Groettrup M. Prostaglandin E2 inhibits IL-23 and IL-12 production by human monocytes through down-regulation of their common p40 subunit. $\mathrm{Mol}$ Immunol 2013:53:274-82. 